\title{
STY1 and STY2 promote the formation of apical tissues during Arabidopsis
} \section{gynoecium development}

\author{
Sandra Kuusk ${ }^{1, \dagger}$, Joel J. Sohlberg${ }^{1}$, Jeff A. Long ${ }^{2}$, Ingela Fridborg ${ }^{1, \star}$ and Eva Sundberg ${ }^{1, \dagger}$ \\ ${ }^{1}$ Department of Physiological Botany, Evolutionary Biology Centre, Uppsala University, Villavägen 6, S-752 36 Uppsala, Sweden \\ ${ }^{2}$ California Institute of Technology, Division of Biology 156-29, 1200 East California Boulevard, Pasadena, CA 91125, USA \\ *Present address: Department of Plant Biology, Genetic Centre, Swedish University of Agricultural Sciences, Box 7080, S-750 07 Uppsala, Sweden \\ †Authors for correspondence (e-mail: sandra.kuusk@ebc.uu.se and eva.sundberg@ebc.uu.se) \\ Accepted 10 July 2002
}

\section{SUMMARY}

Gynoecium ontogenesis in Arabidopsis is accomplished by the co-ordinated activity of genes that control patterning and the regional differentiation of tissues, and ultimately results in the formation of a basal ovary, a short style and an apical stigma. A transposon insertion in the STYLISHI (STY1) gene results in gynoecia with aberrant style morphology, while an insertion mutation in the closely related STYLISH2 (STY2) gene has no visible effect on gynoecium development. However, sty1-1 sty2-1 double mutant plants exhibit an enhanced sty1-1 mutant phenotype and are characterized by a further reduction in the amount of stylar and stigmatic tissues and decreased proliferation of stylar xylem. These data imply that STY1 and STY2 are partially redundant and that both genes promote style and stigma formation and influence vascular development during Arabidopsis gynoecium development.
Consistently, STY1 and STY2 are expressed in the apical parts of the developing gynoecium and ectopic expression of either $S T Y 1$ or $S T Y 2$ driven by the CaMV 35S promoter is sufficient to transform valve cells into style cells. STY1::GUS and STY2::GUS activity is detected in many other organs as well as the gynoecium, suggesting that STY1 and STY2 may have additional functions. This is supported by the sty1-1 sty2-1 double mutants producing rosette and cauline leaves with a higher degree of serration than wild-type leaves. STY1 and STY2 are members of a small gene family, and encode proteins with a RING fingerlike motif. Double mutant analyses indicate that STY1 genetically interacts with SPATULA and possibly also with CRABS CLAW.

Key words: Arabidopsis thaliana, Gynoecium, Style, STYLISHI

\section{INTRODUCTION}

The seed-bearing female reproductive unit of the angiosperm flower, the gynoecium, is a highly complex organ that differs widely in form between species. Despite the extensive differences in the overall morphology of gynoecia of different angiosperms, a set of common structures, characterized by cell types with unique properties, can be distinguished. At maturity, most gynoecia are composed of a basal ovary, which encloses the ovules, a style, and an apical stigma to which the pollen grains adhere. The stylar region varies in length and appearance in association with species specific pollination strategies.

In Arabidopsis, the gynoecium consists of two congenitally fused carpels. On top of a basal, bilocular ovary a short style places the stigmatic surface in close contact with the anthers allowing self-pollination to occur. Four main parallel vascular bundles run longitudinally through the Arabidopsis ovary and ovules arise from placental tissue along the margins of the site of carpel fusion. A central transmitting tract, composed of polysaccharide-rich tissue that directs pollen tube growth, spans the entire organ. Following fertilization, a prominent elongation of the ovary precedes dehiscence and dispersal of the mature seeds. The morphology of the Arabidopsis gynoecium has been described in detail elsewhere (Okada et al., 1989; Smyth et al., 1990; Sessions and Zambryski, 1995).

The sequential process of gynoecium organogenesis relies upon the coordinated activity of a large set of genes, but the specification of carpel identity in the central zone of the floral meristem is established by the action of a single gene, the MADS-box gene AGAMOUS (AG) (Bowman et al., 1989; Bowman et al., 1991; Mizukami and Ma, 1992). In the subsequent developmental processes, $A G$ function is absolutely required only for the formation of the carpel wall, as style, stigma, transmitting tract and placental tissues can appear in the absence of $A G$ activity (Bowman et al., 1991). Other genes act to promote the formation of these characters.

ETTIN (ETT) and PINOID (PID) appear to be involved in the regulation of the apical-basal patterning of the developing gynoecium (Sessions et al., 1997; Bennett et al., 1995) and recent data demonstrate links between the phytohormone auxin and ETT and PID activities (Nemhauser et al., 2000; Christensen et al., 2000; Benjamins et al., 2001). The establishment of the internal/external polarity of the gynoecium, however, involves members of the KANADI and $Y A B B Y$ gene families, which act by specifying abaxial identity (Eshed et al., 2001; Siegfried et al., 1999; Eshed et al., 1999).

A number of mutants with defects restricted to specific 
tissues within the gynoecium have been isolated (reviewed by Ferrándiz et al., 1999) and their corresponding genes likely participate in the regulation of the differentiation and organization of these tissues rather than in the general patterning of the gynoecium. Phenotypic analyses of plants mutant for SPATULA (SPT), TOUSLED (TSL) and CRABS $C L A W$ (CRC) suggest that SPT and TSL promote the development of apical margin tissues such as style, stigma and transmitting tract while $C R C$ promotes style formation and regulates the radial and longitudinal expansion of the gynoecium (Alvarez and Smyth, 1999; Roe et al., 1997; Bowman and Smyth, 1999). Differentiation and elongation of the valve is regulated by the FRUITFUL (FUL) (Gu et al., 1998) and SHATTERPROOF genes (SHP) (Liljegren et al., 2000).

Even though our understanding of the mechanisms underlying the cellular organization of the complex gynoecial structure has increased substantially during the last few years, a large set of genes with regulatory functions in gynoecium ontogenesis likely remains to be identified. This work describes the functional characterization of two new genes that promote the formation of apical tissues during gynoecium development.

We use a reverse genetics approach to identify new genes that regulate different aspects of development in Arabidopsis. In a database search we identified STYLISHI (STYI) and STYLISH2 (STY2) as members of the SHI gene family (Fridborg et al., 2001), and screened for insertion mutations in these genes. The phenotypic properties of styl-1 single mutants, of sty1-1 sty2-1 double mutants and of transgenic plants expressing either STY1 or STY2 ectopically, imply that $S T Y 1$ and STY2 have partially redundant functions and that they are required specifically for the promotion of style and stigma formation and for the establishment of style architecture.

\section{MATERIALS AND METHODS}

\section{Plant material and genetics}

Arabidopsis thaliana ecotype Columbia was used unless otherwise indicated. Columbia wild-type seeds and $c r c-1$ mutant seeds were provided by the Arabidopsis Biological Research Center (ABRC, Columbus, USA). spt-2 seeds were kindly provided by John Bowman. Seeds were surface sterilized as described previously (Fridborg et al., 1999), cold treated for 2-3 days and cultured in cool white fluorescent light at $20-22^{\circ} \mathrm{C}$ under long day conditions (18 hours light, 6 hours dark). sty1-1 sty2-1, sty1-1 crc-1 and sty1-1 spt-2 double mutants were generated by cross-fertilization of homozygous single mutants. Double mutants were identified among the $\mathrm{F}_{2}$ segregants. Floral stages are defined according to Smyth et al. (Smyth et al., 1990).

\section{Mutant screening}

The SLAT collection (Tissier et al., 1999) was PCR screened using STY1 or STY2 gene-specific primers (primers available upon request), in combination with the transposon primers dSPM1 and dSPM11 according to the SLAT manual (http://nasc.nott.ac.uk/info/ slat_infol.html). Identities of PCR products were verified by sequencing and by southern blot analysis, according to standard procedures. Mutants were backcrossed to wild type and the $\mathrm{F}_{2}$ progenies segregated for a single insert in both STY1 and STY2.

\section{STY1 and STY2 constructs and plant transformation}

For GUS reporter gene constructs, a $2 \mathrm{~kb}$ SalI/BamHI fragment of the
STY1 promoter and a $2.1 \mathrm{~kb} X h o \mathrm{I} / B g l \mathrm{II}$ fragment of the STY2 promoter, respectively, were ligated into the pBI101.3 binary vector (Clontech, Palo Alto, CA) upstream of the uidA open reading frame (ORF) (Jefferson, 1987). Constructs were transformed into the Agrobacterium tumefaciens strain GV3101 containing the helper plasmid pMP90. The $S T Y 1$ cDNA was obtained by fusing a $0.67 \mathrm{~kb}$ B $g$ III/DraII fragment from the STY1 genomic clone to a $0.77 \mathrm{~kb}$ DraII STY1 EST fragment (GenBank accession number T88542) in the pBluescriptIISK+/- vector (Stratagene, La Jolla, CA). The full-length coding sequence was then PCR amplified. The STY2 coding sequence was amplified by reverse transcriptase (RT)-PCR using the Access RTPCR system (Promega). PCR products were ligated into the pCR2.1 TOPO-TA vector (Invitrogen, Leek, the Netherlands) and sequenced. Verified products were cloned into the pHTT202 vector (Elomaa et al., 1993), downstream of the CaMV $35 \mathrm{~S}$ promoter in sense orientation. Constructs were introduced into the Agrobacterium tumefaciens strain C58::pGV2260 by triparental mating. All plasmids were introduced into Arabidopsis by Agrobacterium tumefaciens mediated transformation according to the method of Bechtold et al. (Bechtold et al., 1993).

\section{In situ hybridization and histochemical staining}

Fixation, probe synthesis and in situ hybridization was performed as described previously (Long et al., 1996). A detailed protocol can be found at http://www.its.caltech.edu/ plantlab/protocols/ nonradinsitu.html. STY1 sense and antisense transcripts were generated from the $S T Y 1$ cDNA cloned into the pBluescriptII SK+/vector using the T3 and T7 RNA polymerase, respectively, and labeled with digoxigenin-11-UTP. For the antisense probe, a $368 \mathrm{bp}$ fragment containing the $3^{\prime}$ end of the STY1 cDNA was used, whereas the sense probe was generated from a $1422 \mathrm{bp}$ fragment comprising the whole STY1 ORF.

Histochemical staining for GUS activity was performed according to the method of Jefferson (Jefferson, 1987). Ferri/ferrocyanide was used in the staining solution.

\section{Scanning electron microscopy (SEM)}

Fixation, drying and viewing was performed as described by Fridborg et al. (Fridborg et al., 1999).

\section{Transmission electron microscopy (TEM) and light field microscopy}

Samples were fixed in $2.5 \%$ glutaraldehyde overnight, washed and fixed in $1 \%$ osmium tetroxide for 2 hours. Following washing and dehydration, samples were infiltrated in TAAB812 resin-acetone (1:1) overnight and embedded in silicon plates. Sections were viewed with a transmission electron microscope (Philips, CM10) or with a light microscope (Leica, Leitz DMRX).

\section{Dark field microscopy}

Plant material was fixed, cleared and mounted essentially according to the method of Berleth and Jürgens (Berleth and Jürgens, 1993) except that the incubation in chloralhydrate was exchanged for a 16 hours incubation in $8 \mathrm{~N} \mathrm{NaOH}$ followed by 1 hour in $0.1 \mathrm{M}$ potassium phosphate buffer, $\mathrm{pH}$ 7.5. Samples were viewed with a light microscope (Leica, Leitz DMRX).

\section{RESULTS}

\section{Identification of the STY1 and STY2 genes}

We have previously reported on the identification and characterization of the gain-of-function mutant short internodes (shi) that displays a dwarf phenotype typical for mutants defective in the biosynthesis of the plant hormone gibberellin (GA) (Fridborg et al., 1999). Cloning of the SHI gene revealed 
Fig. 1. Gene organization of $S T Y 1$ and $S T Y 2$ and locations of the transposon insertions in sty $1-1$ and sty2-1. Rectangles correspond to translated regions and lines depict transcribed but untranslated regions and introns. The sequence elements encoding the RING and IGGH domains are boxed. Cysteines and histidines that constitute putative zinc ligands are in bold and enlarged. The putative NLS of each encoded protein is underlined. Triangles denote transposon insertions. All lines and boxes are drawn to scale.

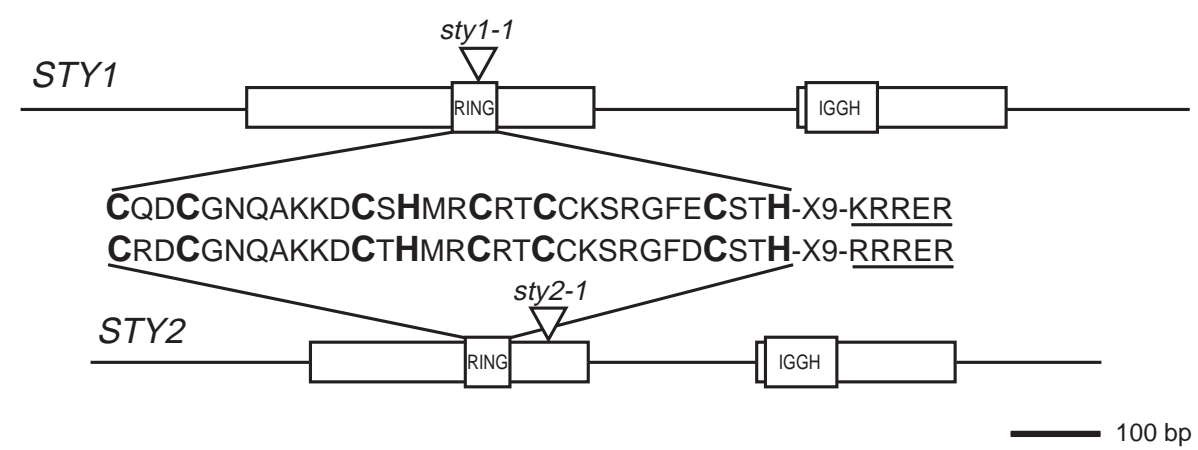

that it encodes a putative zinc finger protein, suggested to act as a repressor of GA responses (Fridborg et al., 1999). In a search through the Arabidopsis Genome Initiative (AGI) database (http://www.arabidopsis.org/ agi.html) for genes similar to $S H I$ and its related gene LATERAL ROOT PRIMORDIUM 1 (LRPI) (Smith and Fedoroff, 1995), we identified STYLISH1 and STYLISH2 (STY1 and STY2; GenBank IDs: At3g51060 and At4g36260, respectively; note that the annotated protein At3g51060 is shorter than STY1, probably due to a sequencing error that has introduced a premature stop codon). $S T Y 1$ and $S T Y 2$ have previously been referred to as $S H I$ RELATED SEQUENCE 1 and 2 (SRS1 and SRS2) (Fridborg et al., 2001). STY1 maps to the bottom of chromosome 3 close to the marker MUR_1, and STY2 maps to the bottom of chromosome 4 close to marker FAH1.

Database searches revealed six additional Arabidopsis paralogs: $S R S 3$ to $S R S 8$ with the following GenBank IDs: At2g21400 (SRS3), At2g18120 (SRS4), At1g75520 (SRS5), At3g54430 (SRS6), At1g19790 (SRS7) and At5g33210 (SRS8). Together these genes constitute the SHI gene family (Fridborg et al., 2001).

Database searches served also to identify putative homologs in other angiosperms (Fridborg et al., 2001). No genes with recognizable sequence similarities to the SHI-like genes were found in organisms other than plants, suggesting that this class of genes is unique to plants.

Comparisons between the STY1 and STY2 cDNAs and their respective genomic sequences showed that the STY1 gene consists of two exons and encodes a putative protein of 370 amino acids with a calculated molecular mass of $38.4 \mathrm{kDa}$. The STY2 gene has two exons and encodes a 322 amino acid protein with a predicted mass of $34.1 \mathrm{kDa}$. A comparison of the deduced amino acid sequences of STY1 and STY2 with those of other members of the SHI family revealed two main regions with a high degree of sequence identity. A cysteine/histidine rich stretch, with the consensus sequence $\mathrm{C}-\mathrm{X}_{2}-\mathrm{C}-\mathrm{X}_{7}-\mathrm{C}-\mathrm{X}-\mathrm{H}-$ $\mathrm{X}_{2}-\mathrm{C}-\mathrm{X}_{2}-\mathrm{C}-\mathrm{X}_{7}-\mathrm{C}-\mathrm{X}_{2}-\mathrm{H}$, or $\mathrm{C} 3 \mathrm{HC} 3 \mathrm{H}$ (amino acids 144-174 and 94-124, in STY1 and STY2 respectively), is present in all family members and is $90 \%$ identical between STY1 and STY2 (Fig. 1). This consensus sequence is similar to the consensus of the zinc binding C3HC4 RING finger motif (Freemont, 1993; Lovering et al., 1993), which generally mediates proteinprotein interactions in numerous otherwise unrelated proteins in different eukaryotes (reviewed by Borden, 2000). A putative nuclear localization signal (NLS) (Boulikas, 1994; LaCasse and Lefebvre, 1995) is located immediately downstream of the putative RING domains of STY1 and STY2 (Fig. 1).
A second sequence element, the IGGH domain, present in all SHI family members (Fridborg et al., 2001), is located distally to the putative RING finger (amino acids 238-285 and 197-245 in STY1 and STY2 respectively). This domain shows no distinguishable sequence similarity to any functionally characterized motif. STY1 and STY2 are 67\% identical over this region. Overall, STY1 and STY2 share $43 \%$ amino acid identity.

\section{A transposon insertion in STY1 disrupts style formation}

We screened all available T-DNA and transposon collections to identify knockout alleles of STY1 and STY2. In the SLAT transposon collection (Tissier et al., 1999) we identified one $\mathrm{d}$ Spm transposon insertion in each of the STY1 and STY2 ORFs. The mutant alleles were designated styl-1 and sty2-1, respectively. In sty $1-1$, the transposon is inserted at position 484 of the STY1 coding sequence, in the sequence element encoding the putative zinc finger (Fig. 1). sty2-1 harbors a transposon insertion at position 483 of the coding region, 82 bases upstream of the STY2 $5^{\prime}$ intron splice site (Fig. 1).

The homozygous sty2-1 mutant plants grown under long day conditions show no apparent morphological differences from wild type except for slightly more serrated rosette and cauline leaves (data not shown). sty 1-1 mutant plants grown under the same conditions are also essentially wild-type-like, but exhibit some morphological aberrations that are restricted to the apical parts of the gynoecium.

The wild-type Arabidopsis gynoecium emerges as a hollow cylinder in the central position of the flower. At stage 10 of flower development it closes at its apical end and the medial septum forms (Smyth et al., 1990). During stage 11, the style epidermis cells differentiate to become morphologically distinct from the ovary and stigmatic papillae develop at the flat upper surface of the gynoecium (Smyth et al., 1990). Stage 12 wild-type style epidermal cells have a characteristically ridged surface (Fig. 5Q) and are arranged in ordered longitudinal cell files (Fig. 2A, Fig. 5B) (Smyth et al., 1990).

Mature sty 1-1 styles exhibit a slight depression, mostly in the medial plane (Fig. 2B), and are generally wider (Fig. 2C,D) than their wild-type counterparts (Fig. 2A). Some style cells expand abnormally and adopt an uncoordinated growth pattern, often resulting in the presence of style epidermal cells on the apical margins of the gynoecium (Fig. 2C,D), where the wild type forms stigmatic papillae. This, in turn, results in an abnormal growth pattern of sty 1-1 papillar cells which, instead of pointing upwards, tend to sprawl in all directions (Fig. 2C). 
Fig. 2. Scanning electron micrographs of gynoecia of wild type, the sty 1-1 mutant and the styl-1 sty2-1 double mutant. (A) Medial view of a stage 13 wildtype gynoecium. Stigma, style, the two valves and the abaxial replum are indicated. (B-D) Medial views of styl-1 single mutant gynoecia of increasing age: (B) stage 12, (C) stage 13, (D) stage 15 . Note the style cells in apical positions (C,D, arrowheads). (E) Top view of a stage 12 styl-1 sty2-1 double mutant gynoecium. Arrowhead points to misplaced apical and adaxial style cells. (F-G) Stage 13 sty $1-1$ sty2-1 double mutant gynoecia that have developed short horn-like protrusions and produced clusters of stigmatic tissue ( $\mathrm{H}$, arrowhead). Arrowhead in $\mathrm{F}$ indicates the adaxially formed style cells. Scale bar: $100 \mu \mathrm{m}$.
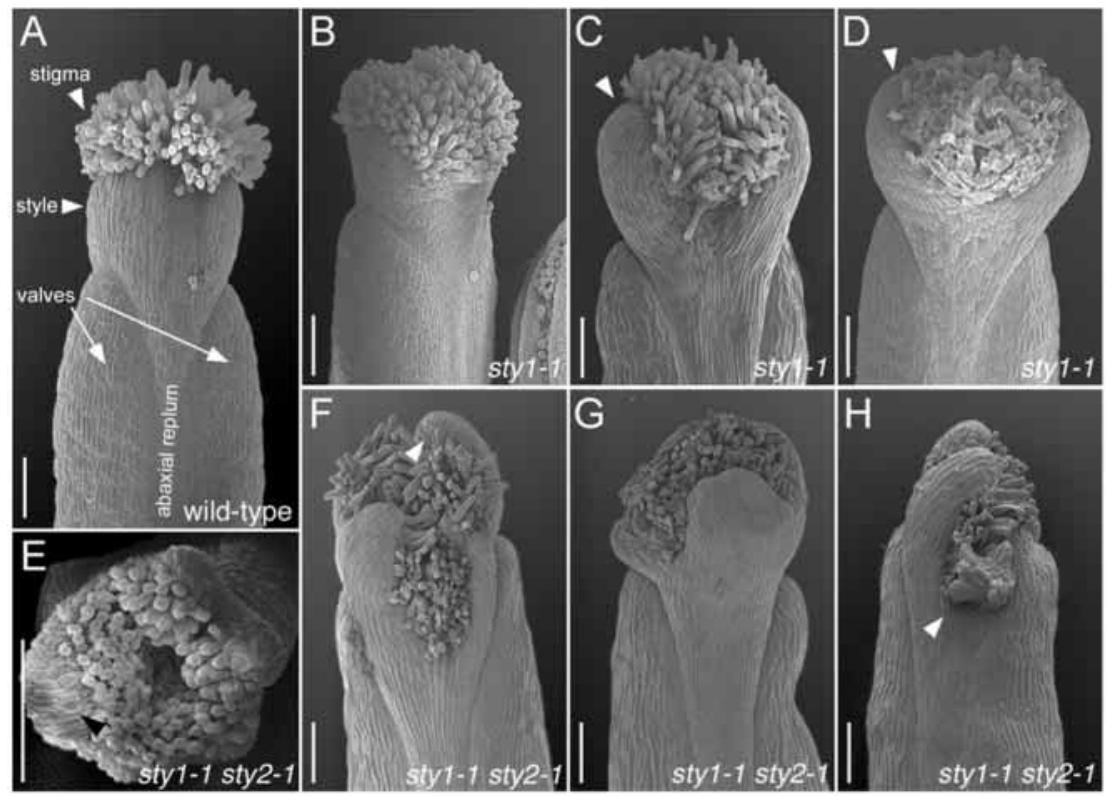

The apical abaxial (outer) replum is most often broader in styl1 gynoecia (Fig. 2C,D) than in wild-type gynoecia. Stomata development and distribution in the mutant styles is similar to that in wild type. Most styl-1 mutants exhibit a minor reduction in fertility as the uppermost ovules of each silique often abort, resulting in a silique that narrows apically. A minority of the styl-1 mutant gynoecia is nearly wild-type-like and occasionally, gynoecia with more severe morphological defects are formed, either in the most basal or apical parts of the inflorescence or after conditions of water deficit (data not shown).

\section{The sty1-1 mutant phenotype is enhanced in sty2-1 mutant background}

We tested if STY1 and STY2 have redundant functions by creating a sty $1-1$ sty2-1 double mutant. Strikingly, all gynoecia of the double mutant plants display an enhanced styl-1 phenotype and develop an aberrant style with more pronounced defects compared to those seen in the styl-1 single mutant.

In more detail, gynoecia of stage 11 styl-1 sty2-1 flowers have a central narrow cavity surrounded by style cells growing in three to five bulging lobes (Fig. 2E). During stages 11-13, some, or all, lobes elongate to short protrusions (Fig. 2F,G,H). In certain gynoecia this causes the formation of ridged epidermal style cells not only apically (as in the sty $1-1$ mutant) but also adaxially, on the inside of the gynoecium (Fig. 2F). Stigmatic papillae develop along the edges of each lobe and from the adaxially positioned style cells (Fig. 2F,G,H). Sometimes, the stigmatic papillae are clustered together in lump-like structures (Fig. 2H). The amount of stylar and stigmatic tissues in each gynoecium of the double mutant is markedly reduced compared to wild type and to the styl-1 single mutant. The septum is frequently absent from, or reduced in, the apical parts of the ovary. The broad abaxial replum observed in styl-1 single mutants, is distinct also in sty1-1 sty2-1 double mutants. Despite the dramatic change in style appearance, the double mutant plants display no significant reduction in fertility compared to the sty $1-1$ mutant.
In addition to the carpel defects, the rosette and cauline leaves of the sty1-1 sty2-1 double mutant plants are more serrated than their wild-type counterparts and the sty2-1 single mutant leaves (Fig. 3). The difference from wild type is most distinct before the leaves are fully expanded.

\section{Vascular development is altered in gynoecia of sty1-1 and sty1-1 sty2-1}

The wild-type Arabidopsis gynoecium contains four vascular bundles, two lateral and two medial, that run parallel to the ovary axis (Fig. 4A). The lateral veins terminate in the valves just below the ovary/style junction, while the medial veins bifurcate at this point and connects with fans of xylem elements along the medial side of the style (Fig. 4A).

We studied cleared gynoecia using dark-field microscopy and found that the organization of the medial veins in gynoecia of sty 1-1 and sty 1-1 sty2-1 was altered compared to wild type, whereas the lateral bundles differentiated and terminated normally. In gynoecia of sty $1-1$ we frequently noted a basalized point of medial vein bifurcation and slightly reduced xylem fans that were located on the lateral, rather than along the medial, sides of the style (Fig. 4B). In the sty1-1 sty2-1 double

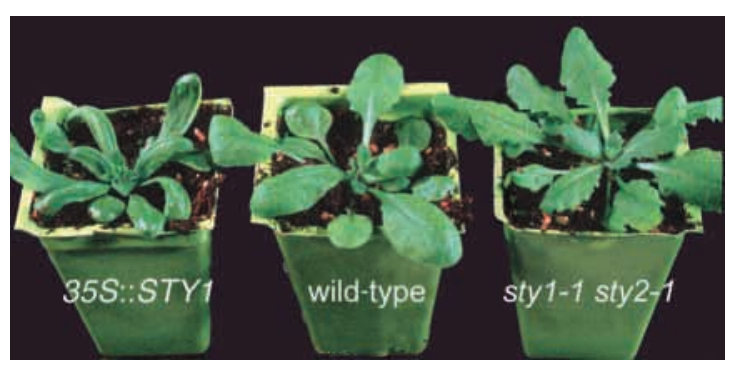

Fig. 3. Rosette leaf morphology of 35S::STY1, wild type and sty1-1 sty2-1 double mutant plants. Plants overexpressing STY1 or STY2 typically produce narrow, non-serrated, epinastic leaves (left). sty 1-1 sty2-1 double mutant plants develop leaves with increased serration (right) compared to wild-type leaves (center). 


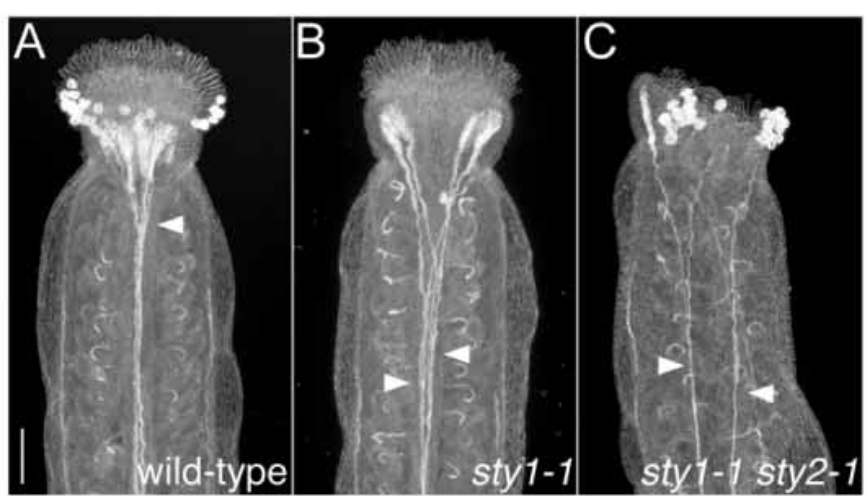

Fig. 4. Vascular patterning in wild type, sty 1-1 and sty1-1 sty2-1 gynoecia at anthesis. In each gynoecium, the points of bifurcation of the medial veins are indicated with arrowheads. Medial views of (A) a wild-type gynoecium showing medial veins bifurcating just below the style and medial xylem fans extending along the medial side of the style; (B) a sty 1-1 gynoecium in which the point of bifurcation of the medial veins is basalized and the medial xylem fans are located laterally on the style; (C) a sty 1-1 sty2-1 gynoecium in which the point of bifurcation of the medial veins is basalized and medial xylem fan formation is greatly reduced. Note that pollen grains appear as white spots. Scale bar: $100 \mu \mathrm{m}$.

mutant, the stylar xylem fans were further reduced or even absent (Fig. 4C). A basalized point of medial vein bifurcation, similar to that observed in the sty $1-1$ single mutant, was also evident (Fig. 4C). We could not detect any abnormalities in the vascular patterning in the sty2-1 single mutant.

\section{Constitutive expression of STY1 and STY2 causes ectopic style cell formation}

To study the effects of ectopic expression of STY1 and STY2 on gynoecium development we generated transgenic plants in which the ORFs of STY1 and STY2 were expressed under the control of the CaMV 35S promoter (35S::STY1, $35 S:: S T Y 2)$. Plants from 11 independent 35S::STY1 lines and 7 independent $35 S:: S T Y 2$ lines displayed the same phenotypic properties.

Compared to wild type (Fig. 2A, Fig. 5B), mature styles of the transgenic $35 S:: S T Y 1$ and $35 S:: S T Y 2$ plants are shorter and more blunt in shape (Fig. 5A) and the style epidermal cells are disordered and irregularly shaped and sized (Fig. 5C). In the upper, lateral parts of the ovary, where valve cells with a flat cuticle normally form (Fig. 5K,P), ridged, style-like epidermal cells are present (Fig. 5A,D,L,M). These cells differentiate simultaneously with the true style cells of the transgenic plants (Fig. 5C,R) and display obvious similarities with wild-type style epidermal cells (Fig. 5Q). We therefore conclude that they are ectopically formed style cells. Studies of cross sections of 35S::STY1 gynoecia showed that the change in cell identity, from valve to style cells, occurs in both abaxial (Fig. 5L,M) and adaxial positions (Fig. 5L,N); alternatively, the inner epidermal cells, in contrast to their wild-type counterparts (Fig. $5 \mathrm{G})$, are striate. The cells in the immediate vicinity to the ectopic style cells display characteristics of both style and valve epidermal cells (Fig. 5D,L), suggesting that the valve to style cell transition occurs gradually.

At anthesis, the wild-type valves are clearly distinguishable
STY1 and STY2 promote gynoecium development

from the style and the replum (Fig. 2A). The differentiated wild-type ovary wall consists of six cell layers. From the abaxial to the adaxial side of the valve, four distinct cell types are recognized: an outer epidermis, three layers of chlorenchyma cells, a layer subjacent to the inner epidermis with longitudinally elongated cells, and an inner epidermis composed of radially elongated cells (Fig. $5 \mathrm{H}$ ).

In $35 S:: S T Y 1$ and $35 S:: S T Y 2$ valves, all cell layers in the area between the ridged style-like outer and inner epidermal cells are similar in size and shape to the epidermal cells (Fig. 5I). In some gynoecia extra cell layers of irregularly expanding chlorenchyma cells flanking the ectopic style cells can be noted (Fig. 5I).

The full-grown siliques of $35 S:: S T Y 1$ or $35 S:: S T Y 2$ plants are shorter than their wild-type counterparts and the upper third, or half, of each silique is often compressed and slightly bent (Fig. 5F). This is probably due to the failure of the ectopic style cells to expand longitudinally to the same extent as the surrounding true valve cells.

The ectopically produced style cells form a continuum with the true style cells so that no valve margins are defined at the valve/style boundary. Consequently, no proper dehiscence zone seems to form in this area and the valves often remain partly attached after seed dispersal (Fig. 5J). The formation of ectopic style cells in the transgenic plants is restricted to valves. In severely affected lines, petals are shorter and narrower than wild type and fertility is much reduced because of poorly elongated stamens and underdeveloped anthers with low pollen production (Fig. 5E).

The transformant plants show a range of additional traits including epinastic, lancet shaped rosette leaves (Fig. 3), reduced stem elongation and late flowering during short day conditions (data not shown). In these respects, the 35S::STY1 and $35 S:: S T Y 2$ plants are identical to the shi gain-of-function mutant (Fridborg et al., 1999). However, shi does not produce ectopic style cells.

\section{Constitutive expression of STY1 and STY2 affects vascular patterning in the gynoecium}

Vascular formation and distribution in gynoecia of plants overexpressing $S T Y 1$ or $S T Y 2$ differ from wild type. The lateral veins of $35 S:: S T Y 1$ or $35 S:: S T Y 2$ gynoeica often terminate into thin fan-like structures, similar to those formed by the medial veins that differentiates in the style (Fig. 5O,S). In addition, discontinuous xylem strands, parallel to the lateral bundles or bent towards the ectopically produced style cells, that produce fan-like structures at their apical ends are often present (Fig. $5 \mathrm{O}, \mathrm{S})$. The medial veins regularly poly-bifurcate and produce massive xylem fans, which, like wild-type fans, extend along the medial face of the style (Fig. 5O,S).

\section{STY1 is expressed in the developing gynoecium until anthesis}

STY1 expression in wild-type floral tissues was monitored using in situ hybridization.

Expression of STY1 was detected throughout the youngest flower primordium (P1; Fig. 6A). By stage 1, the STY1 expression started to relocalize towards the regions of the presumptive sepal anlagen and remained in sepal primordia until just after their emergence (Fig. 6A,B). Early expression of STY1 was also detected on the abaxial side of the young 
Fig. 5. Phenotypes associated with $35 S:: S T Y 1$ and $35 S:: S T Y 2$ plants. For simplicity, all illustrations show $35 S:: S T Y 1$ plants. 35S::STY2 plants are identical to $35 S:: S T Y 1$ plants in all aspects shown. (A) Medial view of a stage $1335 S::$ STY1 gynoecium. Regions with ectopic epidermal style cells are bordered by a white line. (B) Close up of wild-type style cells. (C) $35 S:: S T Y 1$ style cells. Close up of upper section indicated in A. (D) Close up of lower section indicated in A that displays the border between valve cells and ectopic style cells in a $35 S:: S T Y 1$ valve. (E) Poorly developed anthers (arrowheads) and narrow petals of a $35 S:: S T Y 1$ flower. (F) Lateral view of a $35 S:: S T Y 1$ silique. (G) Inner valve epidermal cells.

(H) Transverse section of a wildtype valve. (I) Transverse section of a $35 S:: S T Y 1$ valve. Double headed arrow indicates the region of ectopic style cell formation. A mass of overproliferating chlorenchyma cells is seen above the area with ectopic style cells. (J) $35 S:: S T Y 1$ gynoecium with a dehiscing valve still attached to the style along the area where no dehiscence zone is formed (arrow). (K-N; P-R) Transmission
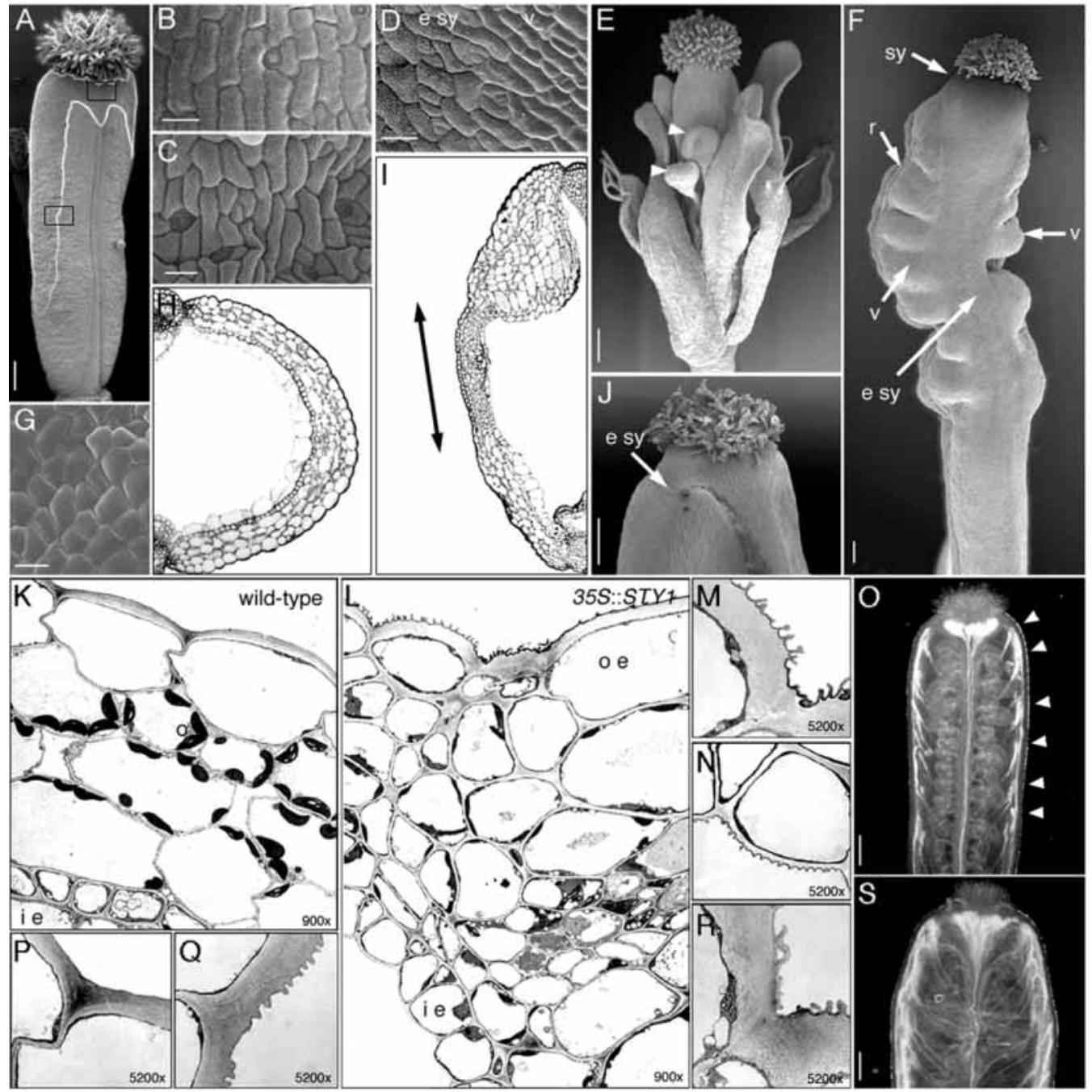
electron micrographs of transverse sections of wild-type and $35 S:: S T Y 1$ valves. (K) Wild-type valve. (L) 35S::STY1 valve. (M) Close up of outer epidermal cells of a $35 S:: S T Y 1$ valve. (N) Close up of inner epidermal cells of a $35 S:: S T Y 1$ valve. (O) Medial view of a $35 S:: S T Y 1$ gynoecia with discontinuous lateral xylem strands (arrowheads) and massive medial xylem fans. (P) Close up of wild-type outer epidermal valve cells. (Q) Close up of wildtype style cells. (R) Close up of $35 S:: S T Y 1$ style cells. (S) Medial view of a 35S::STY1 gynoecia with more severe defects than those in (O). Scale bars: $100 \mu \mathrm{m}$ in A,E,F,J,O,S; $20 \mu \mathrm{m}$ in B,C,D. sy, style; e sy, ectopic style; r, replum; v, valve; o e, outer epidermis; i e, inner epidermis.

floral meristem, in a region suggested to correspond to a highly reduced leaf, or a cryptic bract, subtending the floral bud (Fig. 6C) (Long and Barton, 2000). By floral stage 6, STY1 mRNA was detected in the newly arisen gynoecial primordium (Fig. $6 \mathrm{E})$. As the open-ended gynoecial cylinder elongates vertically (stages 7-10) the STYI expression became restricted to the apical parts of the carpels, extending fully around their circumference (Fig. 6D,F). By stages 11-12, the expression of STY1 in the apical regions of the gynoecium resolved to a zone in the interphase between the style and the stigma (Fig. 6G) from where it declined after stage 13.

Within the gynoecium, STY1 expression was detected in ovule primordia of stage 9 flowers. As the ovules developed, STYI expression became restricted to the epidermis of the developing funiculi, to the outer, but not the inner, integuments (Fig. 6G,H) and to the tip of the nucellus (Fig. 6H). From approximately stage 10, STY1 expression appeared in the cell layer of the septum that faces the ovary (Fig. 6G).
In the embryo STY1 expression was detected in the cotyledon primordia during late globular (Fig. 6I) to mid heart stage (data not shown). STY1 expression transiently appeared in petal and stamen primordia and in the tapetum of the anthers (data not shown). No hybridization was detected with the STYI sense probe (data not shown).

We also studied the $S T Y 1$ promoter activity in transgenic Arabidopsis plants harboring a $2 \mathrm{~kb}$ fragment from the STY1 promoter fused to the uidA gene encoding $\beta$-glucoronidase, GUS (STY1::GUS). The STY1::GUS expression pattern was consistent between 5 independent transgenic lines. In the gynoecium, we detected STY1::GUS expression in a continuous ring in the apical parts of the young emerging gynoecial cylinder (Fig. 6J,M), in accordance with the STYI mRNA distribution pattern determined by in situ hybridization. In the stage 11 gynoecium, STY1::GUS staining accumulated in the style and stigma and two distinct patches of more intense staining, located perpendicular to the plane of medial fusion, 
were observed (Fig. 6N). We also detected STY1 promoter activity in young ovules (Fig. 6J). STY1::GUS activity in style and stigma was low after fertilization.

The loss of STY1 activity causes a mutant phenotype that is limited to the gynoecium. Despite this, STY1::GUS activity was observed in many other tissues. In 8-day-old seedlings, GUS staining was visible in the hypocotyl, in cotyledons and in leaf and root primordia (Fig. 6L). In young developing rosette and cauline leaves $S T Y 1:: G U S$ was primarily expressed in the basal parts of the leaf margins and in hydathodes (Fig. 6K). The STY1 promoter was active in lateral root primordia and in root tips during all stages of the plant life cycle (Fig. 6O,P,Q). STY1::GUS expression was also detected in receptacles (Fig. $6 \mathrm{~J})$ and in stipules (data not shown).

\section{The STY2 promoter is active during late gynoecium development}

A $2.1 \mathrm{~kb}$ STY2 promoter fragment was fused to the uidA gene (STY2::GUS) and the construct was introduced into wild-type plants. The staining pattern was consistent between the 4 independent transformant lines chosen for more extensive analysis.

STY2::GUS activity was absent from the growing carpels until late stage 9 when a weak GUS signal was discerned in the most apical parts of the open-ended gynoecium. From stage 10 , intense GUS staining was seen in the differentiating style and stigmatic tissues (Fig. 7A). GUS expression disappeared from the stigma after fertilization but remained in the style until full silique maturation. STY2 promoter activity was also detected in anthers between stage 7 and stage 10, approximately (Fig. 7A).

In vegetative tissues, the staining pattern from $S T Y 2:: G U S$ construct partly overlapped with that of the STY1::GUS. In 8day-old seedlings, STY2::GUS expression was seen in the shoot apex and in cotyledons (Fig. 7B). Like STY1::GUS, STY2::GUS expression was also visible in leaf primordia (Fig. 7B,C), in stipules (data not shown), in hydathodes of rosette and cauline leaves (Fig. 7C), and in primordia of lateral roots (Fig. 7D,E). $S T Y 2:: G U S$, but not STY1::GUS, activity was detected in developing trichomes (Fig. 7C) of young leaves and in leaf and pedicel attachment sites (Fig. 7A). No expression of the $S T Y 2:: G U S$ transgene was seen in the hypocotyl or primary root tips. Using RT-PCR we detected STY2 transcript in 2week-old seedlings, in roots, in cauline leaves, in flowers and in siliques (data not shown).

\section{Most apical tissues are lost in sty1-1 crc-1 and sty1-1 spt-2 double mutant gynoecia}

Like sty1-1, mutants of $C R C$ and SPT (Fig. 8A,F) have reduced stylar tissue as well as reductions in stylar xylem (Alvarez and Smyth, 1999; Alvarez and Smyth, 2002). To check for possible genetic interactions between STY1 and $C R C$ or $S P T$, respectively, we crossed the corresponding mutants with each other. The $c r c-1$ and spt-2 single mutants are in Landsberg erecta background. As the erecta (er) mutation confers a blunt appearance to the style and causes mature siliques to be shorter and wider than wild-type siliques (Toori et al., 1996) we investigated the influence of the er mutation on the styl-1 crc-1 and styl-1 spt-2 double mutants, with respect to gynoecium development, by studying double mutant plants that were either homozygous for the er mutation or that contained at least one wild-type copy of the $E R$ allele.

styl-1 crc- 1 double mutants exhibit a marked reduction in the amount of stylar and septal tissues and in the number of stigmatic papillae, compared to either single mutant. Patches of stylar tissue (Fig. 8B,C) or style-like structures (Fig. 8D) are formed on each unfused carpel and a few stigmatic papillae are produced (Fig. 8B,C,D). Fertilization most often fails and the gynoecia arrest in growth. Consequently, the double mutants are almost completely sterile. Compared to either single mutant, stylar xylem fan production is reduced, but short fanlike structures are formed in association with the few style cells that are present (Fig. 8E). The lateral veins terminate in fanlike structures in close connection with the medial stylar xylem fans (Fig. 8E). The defects occur independently of a functional $E R$ allele despite the fact that the $\operatorname{crc}$ single mutant phenotype is less pronounced in Columbia background (data not shown).

styl-1 spt-2 double mutants also display severe reductions in stylar and septal tissue and, more conspicuously, a complete loss of stigmatic papillae (the first few flowers may produce small amounts of stigmatic papillae). The apical thirds of the styl-1 spt-2 carpels are typically unfused and the apical carpel margins are lobate (Fig. 8G,H). This alters the entire appearance of the gynoecium as the upper part of the unfused carpels gain a more leaf-like shape at maturity. Stylar tissue is formed at the tips of each carpelloid organ (Fig. 8K) and at the tip of each lateral outgrowth (Fig. 8J). Ovules arise along both fused and unfused margins and the development of the nucellus and the outer and inner integuments initiate normally but the outer integuments seem to grow somewhat abnormally (data not shown) and the double mutant plants are completely sterile.

The lateral veins appear to fuse with the medial ones in the apical part of each carpelloid organ, creating a vascular pattern similar to that in sepals and similar to that in gynoecia of styl1 crc-1 (Fig. 8I,L). In addition, both lateral and medial veins enter some, but not all, lobes where they terminate into fanlike structures (Fig. 8L).

Plants heterozygous for styl-1 and homozygous for spt-2 show an increased loss of stylar and stigmatic tissues and reductions in carpel fusion compared with spt-2 (data not shown) indicating that the level of a functional STY1 protein is critical in the spt-2 mutant background. All phenotypic deviations described for the styl-1 spt-2 double mutants are observed in both $E R$ and er background.

\section{DISCUSSION}

The complex process of gynoecium ontogenesis depends on the regional differentiation of a number of unique cell types and their appropriate organization into distinct tissues. This work demonstrates the involvement of STY1 and STY2 in the formation of style and stigma during gynoecium development in Arabidopsis.

\section{STY1 and STY2 have partially redundant functions during gynoecium development}

Loss of STY1 function causes a decreased production of stylar and stigmatic cells. As all cell types that are present in the wildtype gynoecia are found also in the gynoecia of sty $1-1$ mutants, the role of STYI in gynoecium development seems not to be 


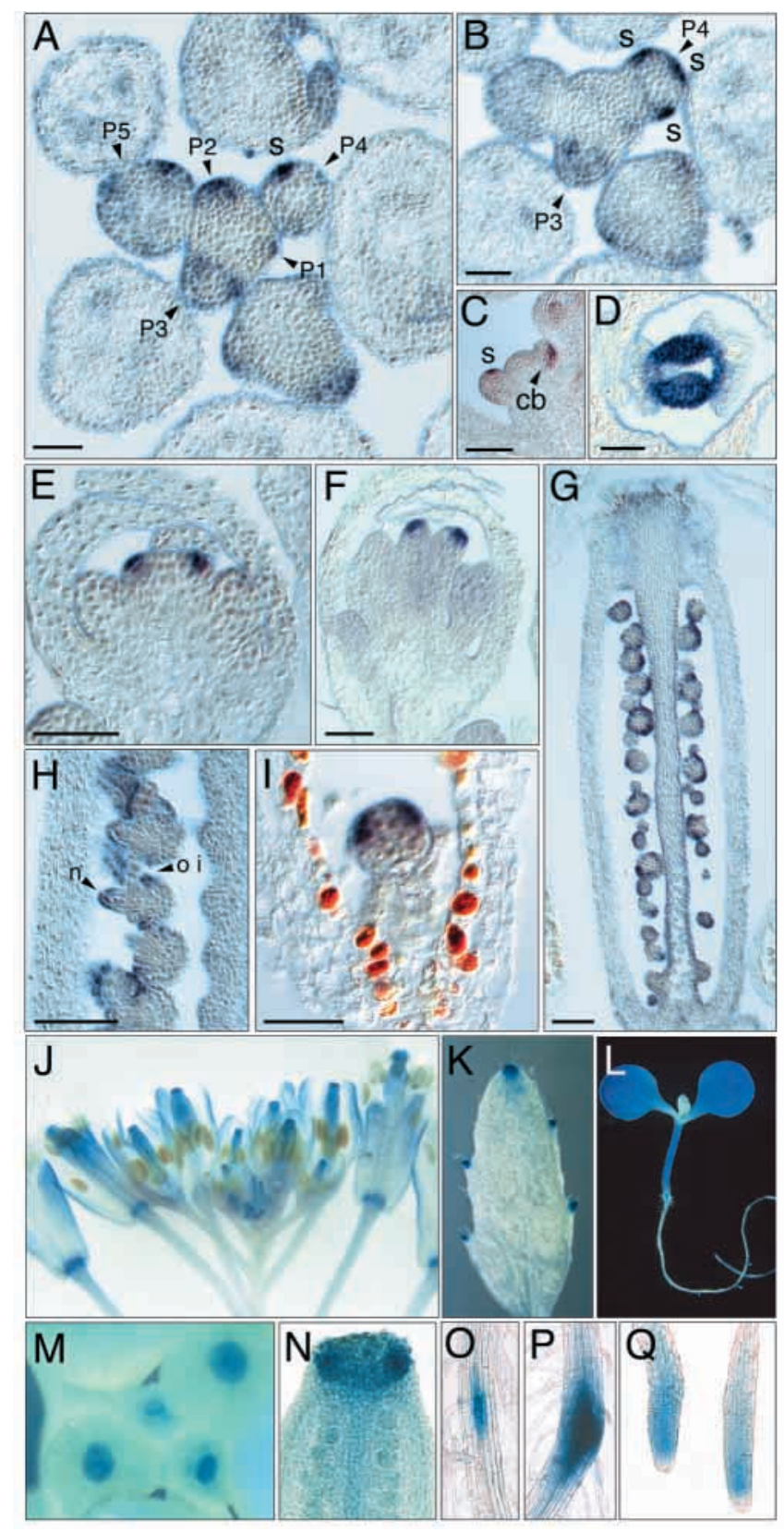

directly related to the control of cellular differentiation. The aberrant style morphology of sty $1-1$ gynoecia more likely results from a partial loss in the control of cell divisions and/or from an irregular and disorganized expansion of the style cells. STY1 may, therefore, act by coordinating the rates and planes of cell divisions and by regulating cell expansion in the developing wild-type style.

Despite the gynoecia of sty2-1 mutants being indistinguishable from wild type, the styl-1 sty2-1 double mutant plants develop gynoecia with much more prominent defects than those found in the styl-1 single mutant. A considerable reduction in the amount of stylar and stigmatic cells in the double mutant, in combination with an uncoordinated growth of the stylar cells, give rise to a style with a severely distorted architecture. Thus, both STY1 and STY2 appear to regulate style and stigma formation and to
Fig. 6. Expression of $S T Y 1$ in wild type determined by in situ hybridization (A-I) and STY1::GUS histochemical staining (J-Q). $(\mathrm{A}, \mathrm{B})$ Transverse consecutive sections of an inflorescence meristem; (A) most apical section. Floral primordia are numbered according to their relative age from P1 to P5. By stage 2 (B) STY1 expression is localized to sepal analgen (s). (C) Longitudinal section of an inflorescence meristem. Expression is seen in sepal primordia and in abaxial parts of the floral meristem (cryptic bract, cb).

(D-F) Expression in the elongating gynoecium. (D) Transverse section of the tip of a stage 8 gynoecium. (E) Longitudinal section of a stage 6 bud. (F) Longitudinal section of a stage 9 flower. Signal is restricted to the apical parts of the gynoecium. (G) Longitudinal section of a stage 12 gynoecium showing expression between the style and the stigma, in outer layers of the septum and in outer integuments. $(\mathrm{H})$ Longitudinal section of young ovules. Expression is seen in outer integuments (o i) and in the tip of the nucellus (n). (I) Longitudinal section of a globular stage embryo. Expression is restricted to cotyledon primordia. Red signals are due to artifacts. (J) Inflorescence. (K) Cauline leaf. (L) 8-day-old seedling. (M) Top view of an inflorescence. Compare with D. (N) Medial view of a stage 12 gynoecium. $(\mathrm{O}, \mathrm{P})$ Emerging lateral root primordia. (Q) Root tips. Scale bars: $50 \mu \mathrm{m}$ in A-F,H; $100 \mu \mathrm{m}$ in G; $40 \mu \mathrm{m}$ in I.

contribute to the establishment of style form. In addition, STY1 appears to fully compensate for the loss of STY2 in the sty2-1 mutant.

The expression patterns of STY1 and STY2 are in accordance with a role for the genes in style development. In situ hybridization and reporter gene experiments showed that STY1 expression appears in the developing gynoecium at floral stage 6 and remains in the apical parts of the gynoecium until anthesis. This pattern directly relates to the sty $1-1$ mutant phenotype and suggests that STY1 acts cell autonomously to regulate style development. The $S T Y 1$ expression data are also consistent with our finding that STY1 functionally substitutes for $S T Y 2$ in the sty2-1 mutant as, until anthesis, STY1 is expressed in all tissues of the gynoecium in which the $S T Y 2$ promoter is active.

As the STY2::GUS expression in the gynoecium does not overlap with the expression of STY1 during early gynoecium development, we hypothesize that this likely limits the ability of STY2 to compensate for the loss of STY1 in styl-1 before stage 10 , even though we cannot exclude the possibility that the $S T Y 2:: G U S$ construct may lack regulatory elements that are required to reflect all $S T Y 2$ expression domains.

CaMV 35S promoter-driven expression of STY1 and STY2 results in the ectopic appearance of style cells where valve cells would normally form. $35 S:: S T Y 1$ or $35 S:: S T Y 2$ plants never produce style cells in any organ but the gynoecium, hence the formation of ectopic style cells in the transgenic plants must be dependent not only on the presence of $S T Y 1$ or $S T Y 2$, but also on other genes that have specific style promoting functions in the gynoecium e.g. CRC and SPT (Bowman and Smyth, 1999; Alvarez and Smyth, 1999).

Since both styl-1 and sty2-1 are very likely to be null mutants, and as not even the sty1-1 sty2-1 double mutant plants completely lack style cells, it appears that neither STY1 nor STY2 is absolutely essential for style cell formation. However, in agreement with the phenotype of $35 S:: S T Y 1$ plants and the early appearance of STY1 expression in apical parts of the gynoecium, STY1 possibly contributes to the specification of style cell identity, redundantly, or in concert with other 


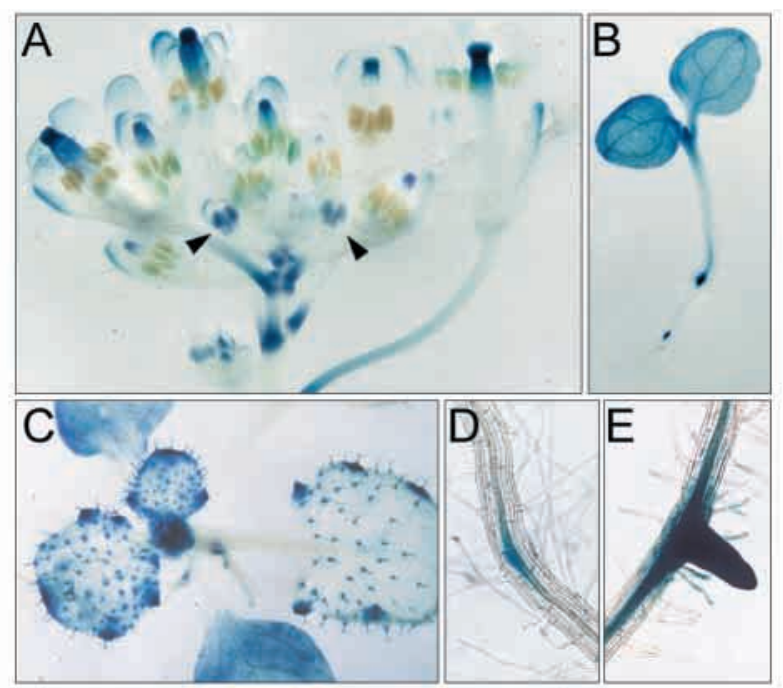

Fig. 7. STY2::GUS localization patterns. (A) Inflorescence showing STY2::GUS staining in style and stigmatic tissues and in anthers (arrowheads). (B) 8-day-old seedling. (C) 2-week-old seedling. Note strong GUS staining in trichomes. $(\mathrm{D}, \mathrm{E})$ Emerging lateral root primordia.

proteins. But, maybe more likely, the increased proliferation of style cells in $35 S:: S T Y 1$ and $35 S:: S T Y 2$ plants may reflect a less coordinated control of cell division and cell expansion, in agreement with the phenotypic effects observed in styl-1 mutants and in styl-1 sty2-1 double mutants.

Taken together, STY1 and STY2 are likely to encode proteins with the same, or very similar, biochemical properties, as ectopic expression of either gene give rise to the same phenotypic effects. Mutant and expression data suggest that the proteins likely promote stylar and stigmatic development by acting in specific, as well as in partly overlapping, regulatory pathways.

\section{Genetic interactions between STY1 and other style and stigma promoting factors}

The formation of apical tissues in the gynoecium is dependent on the activity of several genes. Like styl-1, both $c r c$ and $s p t$ mutants display a reduced production of stylar and stigmatic tissues. In the $c r c-1$ spt-2 double mutant these tissues are even further reduced and the leaf-like carpels are unfused except at the base (Bowman and Smyth, 1999; Alvarez and Smyth, 1999).

The styl-1 crc-1 double mutant plants display a more substantial reduction in stylar, stigmatic, septal and medial xylem tissues compared to either single mutant and are almost completely sterile. Considering the weak phenotype of sty 1-1, a genetic interaction between $S T Y 1$ and $C R C 1$ is possible, as a simple additive effect of both mutations probably would not cause such dramatic decreases in the amount of stylar and stigmatic tissue. The interaction may be indirect, or reflect partially overlapping functions of $S T Y 1$ and $C R C$, but an actual physical interaction between STY1 and CRC is possible as the ring of STYI expression in the apical parts of the gynoecium partially overlaps with the expression domain of $C R C$ in the abaxial carpel epidermis (Bowman and Smyth, 1999) until approximately stage 10 of flower development.
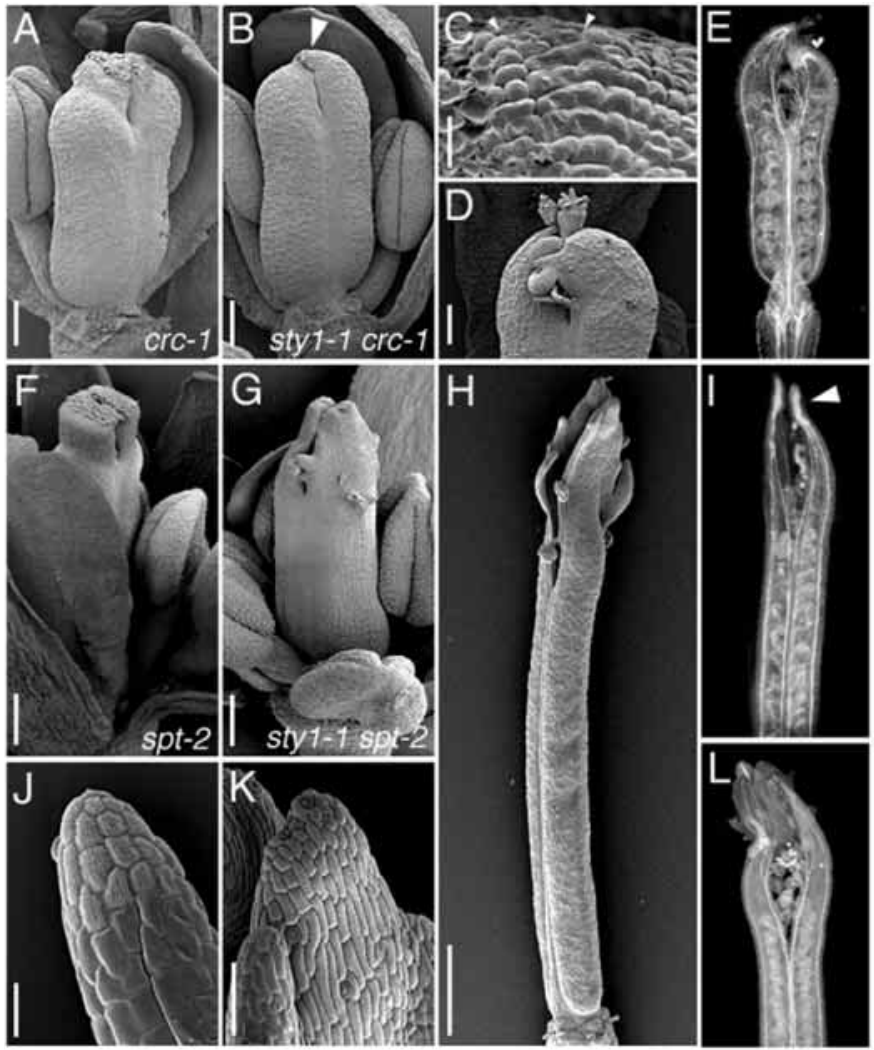

Fig. 8. Phenotypes of styl-1 crc-1 and styl-1 spt-2 double mutants. (A) $c r c-1$ (B) styl-1 crc-1. The style and stigmatic regions are markedly reduced (arrowhead). (C) Enlargement of the region indicated in B. The cells between the arrowheads show style cell characteristics. (D) A sty $1-1$ crc- 1 double mutant gynoecium that has developed two style-like structures with a few stigmatic papillar cells growing from each structure. (E) Medial view of a styl-1 crc-1 gynoecium. (F) spt-2. (G) sty1-1 spt-2. (H) sty1-1 spt-2. Mature silique. (I) Medial view of a styl-1 spt-2 gynoecium. Lateral strands are connected to the medial stylar xylem fans. Arrowhead points to stylar xylem fans produced by both medial and lateral strands. (J) Lateral outgrowth of a sty1-1 spt-2 carpel. Ridged style cells have differentiated at the tip. (K) Apical part of a styl-1 spt-2 gynoecium. Note the absence of stigmatic papillae. (L) Medial view of a sty 1-1 $s p t-2$ gynoecium. Medial and lateral veins enter lobate structures. Scale bars: $100 \mu \mathrm{m}$ in A,B,D,F,G; $20 \mu \mathrm{m}$ in J,C; $50 \mu \mathrm{m}$ in $\mathrm{K}$ and $500 \mu \mathrm{m}$ in $\mathrm{H}$.

In styl-1 spt-2 double mutant gynoecia there is a total absence of stigmatic tissue, except for in the very first flowers. This synergistic effect strongly suggests a genetic interaction between $S T Y 1$ and $S P T$, supported by the partial dominance of styl-1 in spt-2 background. Furthermore, the expression patterns of STY1 and SPT (Heisler et al., 2001) overlap in the medial regions of the apical parts of the gynoecium, from which style and stigma will develop, between stages 6 to 10, and, like STY1, SPT is expressed in ovule primordia and in expanding integuments (Heisler et al., 2001). styl-1 spt-2 double mutants also display an abnormal pattern of medial xylem formation and a marked reduction in stylar and septal tissues.

The phenotypic effects observed in the sty1-1 spt-2 double mutant gynoecia in some aspects resemble those described for 
the $c r c-1 s p t-2$ double mutant. Although the carpels of the styl1 spt-2 double mutants are more fused than those of the $c r c-1$ spt-2 double mutants, the unfused parts in both cases gain a leaf-like appearance and produce similar lateral outgrowths. Furthermore, the vascular patterning in the carpelloid organs is similar between styl-1 spt-2 and $c r c-1$ spt-2 (Alvarez and Smyth, 2002) suggesting that $C R C$ and $S T Y 1$ act in overlapping signal transduction pathways in the apical parts of the developing gynoecium, as supported by the crc-1 sty-1-1 double mutant phenotype.

Apart from regulating valve and replum development, the FUL gene appears to play a role in preventing abnormal style growth as the ful-2 and ful-3 lines develop abnormally elongated styles (Ferrándiz et al., 2000). FUL could therefore impede abnormal style formation through negative regulation of STY1. However, preliminary observations indicate that only additive effects can be detected in the styl-1 ful-2 double mutant (our unpublished data).

\section{STY1 and STY2 affect vascular patterning in the gynoecium}

The reduction of apical tissues in gynoecia of sty $1-1, \operatorname{crc}-1$ and spt-2 single mutants, of crc- 1 spt-2 double mutants (Alvarez and Smyth, 2002), and of double mutants between styl-1 and sty-2, crc- 1 or $s p t-2$, is concomitant with major reductions in stylar xylem and aberrant patterning of the xylem strands. Conversely, in 35S::STY1 and 35S::STY2 plants, an increased proliferation of stylar xylem, as well as a formation of extra xylem strands associated with the ectopically produced style cells, is observed.

Several studies have implicated auxin in vascular development (reviewed by Berleth et al., 2000). Auxin is reported to be predominantly synthesized in apical regions of organs such as leaves and gynoecia, and polar apical-basal flows of auxin appear to influence not only differentiation and patterning of vascular tissues, but also gynoecium morphogenesis (Berleth et al., 2000; Nemhauser et al., 2000).

In wild-type gynoecia treated with inhibitors of polar auxin transport (PAT), the style and internode regions are enlarged at the expense of the ovary and an increased proliferation of stylar xylem can be observed. This is likely a result of auxin accumulation near the source of auxin synthesis, that is, in the stylar regions, and a depletion of auxin in basal parts of the gynoecium (Nemhauser et al., 2000). Accordingly, auxin may be one of the primary signals that triggers the initiation of style development and regulates style cell proliferation. However, the causal relationships between style cell formation and auxin production is not clear as the apical gynoecial tissues also seem to be a main source for auxin synthesis.

If auxin promotes style cell formation, the reduced amounts of style cells and stylar xylem in sty $1-1$ and in sty $1-1$ sty-2 may reflect either a low auxin production, or accumulation, in the apical parts of the gynoecium or a reduced responsiveness to the hormone. Alternatively, if auxin is synthesized in the style cells, the impaired proliferation of stylar xylem observed in sty $1-1$, and in sty $1-1$ combined with sty $2-1, c r c-1$ and $s p t-2$ may be a secondary consequence of reduced auxin production because of a limited number of source cells, that is, style cells.

Correspondingly, the increased production of xylem tissue in $35 S:: S T Y 1$ and $35 S:: S T Y 2$ plants may be due to an increased production or accumulation of auxin, associated with the ectopically produced style cells, or to an enhanced responsiveness to the hormone.

In summary, STY1 and STY2 may promote style formation and influence vascular development by affecting either the levels of auxin in the gynoecium or the transmission of the auxin signal. However, STY1 and STY2 may also regulate style cell proliferation and elongation in a pathway that works independently of auxin.

A derepressible system, involving ubiquitin-targeted proteolytic degradation of one or several putative negative regulators of auxin responses, mediated by the SCFTIR complex, has been described (Gray and Estelle, 2000). As the cysteine-rich region of STY1 and STY2 shows similarities to the consensus sequence of RING finger proteins, and because a majority of the characterized RING finger-containing proteins are associated with ubiquitination (reviewed by Joazerio and Weissman, 2000), STY1 and STY2 could hypothetically be involved in the regulation of auxin responses via targeted protein degradation. However, although a RINGcontaining protein constitutes one of the four subunits in most eukaryotic SCF complexes identified (Callis and Vierstra, 2000), the STY protein is probably not included in the SCFTIR complex. A more likely candidate for the putative RINGcontaining subunit of $\mathrm{SCR}^{\mathrm{TIR}}$ is an Arabidopsis RBX1-like protein.

Furthermore, in a computational analysis of the Arabidopsis proteome Kosarev et al. (Kosarev et al., 2002) identified 387 RING domain proteins with the potential to form RING-type cross brace arrangements. As the last presumptive zinc ligand of STY1 and STY2 is a histidine and not a cysteine, and because the presumptive RING domains of STY1 and STY2 lack certain hydrophobic amino acids, the proteins did not fulfil the criteria used in the analysis and were not among the described RING proteins. Despite that, we cannot exclude the possibility that the zinc finger region of the STY-related proteins constitute a variant of the RING finger domain, or a similar zinc-binding domain, and that the STY proteins may function for example as E3 ligases.

\section{STY1 and STY2 regulate rosette and cauline leaf shape}

The sty1-1 sty2-1 double mutant plants develop rosette and cauline leaves with a higher degree of serration than the leaves of wild type and either single mutant. Conversely, the narrow leaves produced by $35 S:: S T Y 1$ and $35 S:: S T Y 2$ transgenic plants are less serrated than wild-type leaves. We infer, therefore, that STY1 and STY2, in addition to their roles in the gynoecium, act redundantly to regulate the shape of rosette- and cauline leaves. GUS expression driven by both $S T Y 1$ and $S T Y 2$ promoters in leaf primordia, in leaf margins of young developing rosette and cauline leaves and in hydathodes, is in accordance with this hypothesis.

Other mutants defective in gynoecium development, such as tsl (Roe et al., 1993) and leunig (lug) (Liu et al., 1995), display similar defects in leaf morphology. Carpels may be viewed as highly modified leaves or leaf-like structures, which have fused to enclose the developing ovules (Gifford and Foster, 1989). From that perspective, it is possible that the parallel defects in carpel and leaf development observed in several mutants reflect a common evolutionary origin of leaves and carpels. 
We are grateful to John Bowman for helpful comments on the manuscript. We thank Gun-Britt Berglund and Agneta Ottosson for skilful technical assistance, Eva Büren, Marie Lindersson and Caisa Pöntinen for sequencing, and Annette Axén, Stefan Gunnarsson and Gary Wife for help with the microscopy studies. We acknowledge The Wallenberg Consortium North Arabidopsis model organism platform in Uppsala for assistance in the production of transgenic plants and the Sainsbury Laboratory (Norwich, UK) for providing seeds and DNA from the SLAT collection. This work was supported by grants from the Swedish Council for Forestry and Agricultural Research and from the European Community Contract QLG2-CT-1999-00876 (REGIA, to P. Engström).

\section{REFERENCES}

Alvarez, J. and Smyth, D. R. (1999). CRABS CLAW and SPATULA, two Arabidopsis genes that control carpel development in parallel with AGAMOUS. Development 126, 2377-2386.

Alvarez, J. and Smyth, D. R. (2002). CRABS CLAW and SPATULA genes regulate growth and pattern formation during gynoecium development in Arabidopsis thaliana. Int. J. Plant Sci. 163, 17-41.

Bechtold, N., Ellis, J. and Pelletier, G. (1993). In planta Agrobacterium mediated gene transfer by infiltration of adult Arabidopsis thaliana plants. C. R. Acad. Sci. Paris Life Sci. 316, 1194-1199.

Benjamins, R., Quint, A., Weijers, D., Hooykaas, P. and Offringa, R. (2001). The PINOID protein kinase regulates organ development in Arabidopsis by enhancing polar auxin transport. Development 128, 4057-4067.

Bennett, S. R. M., Alvarez, J., Bossinger, G. and Smyth, D. R. (1995). Morphogenesis in pinoid mutants of Arabidopsis thaliana. Plant J. 8, 505520 .

Berleth, T., Mattson, J. and Hardtke, C. S. (2000). Vascular continuity and auxin signals. Trends Plant Sci. 5, 387-393.

Berleth, T. and Jürgens, G. (1993). The role of the monopterous gene in organising the basal body region of the Arabidopsis embryo. Development 118, 575-587.

Borden, K. L. (2000). RING domains: master builders of molecular scaffolds? J. Mol. Biol. 295, 1103-1112.

Boulikas, T. (1994). Putative nuclear localization signals (NLS) in protein transcription factors. J. Cell. Biochem. 55, 32-58.

Bowman, J. L. and Smyth, D. R. (1999). CRABS CLAW, a gene that regulates carpel and nectary development in Arabidopsis, encodes a novel protein with zinc finger and helix-loop-helix domains. Development 126, 2387-2396.

Bowman, J. L., Smyth, D. R. and Meyerowitz, E. M. (1989). Genes directing flower development in Arabidopsis. Plant Cell 1, 37-52.

Bowman, J. L., Smyth, D. R. and Meyerowitz, E. M. (1991). Genetic interactions among floral homeotic genes of Arabidopsis. Development 112, $1-20$.

Callis, J. and Vierstra, R. D. (2000). Protein degradation in signaling. Curr. Opin. Plant Biol. 3, 381-386.

Christensen, S. K., Dagenais, N., Chory, J. and Weigel, D. (2000). Regulation of auxin response by the protein kinase PINOID. Cell 100, 469478 .

Elomaa, P., Honkanen, J., Puska, P., Seppänen, P., Helariutta, Y., Mehto, M., Kotilainen, M., Nevalainen, L. and Teeri, T.H. (1993). Agrobacterium-mediated transfer of antisense chalcone synthase cDNA to Gerbera hybrida inhibits flower pigmentation. Bio/Technology 11, 508-511.

Eshed, Y., Baum, S. F. and Bowman, J. L. (1999). Distinct mechanisms promote polarity establishment in carpels of Arabidopsis. Cell 99, 199-209.

Eshed, Y., Baum, S. F., Perea, J. V. and Bowman, J. L. (2001). Establishment of polarity in lateral organs of plants. Curr. Biol. 11, 1251-1260.

Ferrándiz, C., Pelaz, S. and Yanofsky, M. F. (1999). Control of carpel and fruit development in Arabidopsis. Ann. Rev. Biochem. 68, 321-354.

Ferrándiz, C., Gu, Q, Martienssen, R. and Yanofsky, M. F. (2000). Redundant regulation of meristem identity and plant architecture by FRUITFULL, APETALA1 and CAULIFLOWER. Development 127, 725-734.

Freemont, P. S. (1993). The RING finger. A novel protein sequence motif related to the zinc finger. Ann. N. Y. Acad. Sci. 684, 174-192.

Fridborg, I., Kuusk, S., Moritz, T. and Sundberg, E. (1999). The Arabidopsis dwarf mutant shi exhibits reduced gibberellin responses conferred by overexpression of a new putative zinc finger protein. Plant Cell 11, 1019-1031.
Fridborg, I., Kuusk, S., Robertson, M. and Sundberg, E. (2001). The Arabidopsis protein SHI represses gibberellin responses in Arabidopsis and barley. Plant Phys. 127, 937-948.

Gifford, E. M. and Foster, A. S. (1989). Morphology and Evolution of Vascular Plants. New York: W. H. Freeman.

Gray, W. M. and Estelle, M. (2000). Function of the ubiquitin-proteasome pathway in auxin response. Trends Biochem. Sci. 25, 133-138.

Gu, Q., Ferrándiz, C., Yanofsky, M. F. and Martienssen, R. (1998). The FRUITFULL MADS-box gene mediates cell differentiation during Arabidopsis fruit development. Development 125, 1509-1517.

Heisler, M. G. B., Atkinson, A., Bylstra, Y. H., Walsh, R. and Smyth, D. R. (2001). SPATULA, a gene that controls development of carpel margin tissues in Arabidopsis, encodes a bHLH protein. Development 128, 10891098.

Jeffersson, R. A. (1987). Assaying chimeric genes in plants: The GUS gene fusion system. Plant Mol. Biol. Rep. 5, 387-405.

Joazerio, C. A. P. and Weissman, A. M. (2000). RING finger proteins: mediators of ubiquitin ligase activity. Cell 102, 549-552.

Kosarev, P., Mayer, K. F. X. and Hardtke, C. S. (2002). Evaluation and classification of RING-finger domains encoded by the Arabidopsis genome. Genome Biol. 3, 1-12.

LaCasse, E. C. and Lefebvre, Y. A. (1995). Nuclear localization signals overlap DNA- or RNA-binding domains in nucleic acid-binding proteins. Nucleic Acids Res. 23, 1647-1656.

Liljegren, S. J., Ditta, G. S., Eshed, Y., Savidge, B., Bowman, J. L. and Yanofsky, M. F. (2000). SHATTERPROOF MADS-box genes control seed dispersal in Arabidopsis. Nature 404, 766-770.

Liu, Z. and Meyerowitz, E. M. (1995). LEUNIG regulates AGAMOUS expression in Arabidopsis flowers. Development 121, 975-991.

Long, J. and Barton, K. (2000). Initiation of axillary and floral meristems in Arabidopsis. Dev. Biol. 218, 341-353.

Long, J. A., Moan, E. I., Medford, J. I. and Barton, M. K. (1996). A member of the KNOTTED class of homeodomain proteins encoded by the SHOOT MERISTEMLESS gene of Arabidopsis thaliana. Nature 379, 66-69.

Lovering, R., Hanson, I. M., Borden, K. L. B., Martin, S., O'Reilly, N. J., Evan, G. I., Rahman, D., Pappin, D. J. C., Trowsdale, J. and Freemont, P. S. (1993). Identification and preliminary characterization of a protein motif related to the zinc finger. Proc. Natl. Acad. Sci. USA 90, 2112-2116.

Mizukami, Y. and Ma, H. (1992). Ectopic expression of the floral homeotic gene AGAMOUS in transgenic Arabidopsis plants alters floral organ identity. Cell 71, 119-131.

Nemhauser, J. L., Feldman, L. J. and Zambryski, P. C. (2000). Auxin and ETTIN in Arabidopsis gynoecium morphogenesis. Development 127, 3877 3888.

Okada, K., Komaki, M. K. and Shimura, Y. (1989). Mutational analysis of pistil structure and development of Arabidopsis thaliana. Cell Differ. Dev. 28, 27-37.

Roe, J. L., Nemhauser, J. L. and Zambryski, P. C. (1997). TOUSLED participates in apical tissue formation during gynoecium development in Arabidopsis. Plant Cell 9, 335-353.

Roe, J. L., Rivin, C. J., Sessions, R. A., Feldmann, K. A. and Zambryski, P. C. (1993). The Tousled gene in A.thaliana encodes a protein kinase homolog that is required for leaf and flower development. Cell 75, 939-950.

Sessions, A., Nemhauser, J. L., McCall, A., Roe, J. L., Feldmann, K. A. and Zambryski, P. C. (1997). ETTIN patterns the Arabidopsis floral meristem and reproductive organs. Development 124, 4481-4491.

Sessions, R. A. and Zambryski, P. C. (1995). Arabidopsis gynoecium structure in the wild-type and in ettin mutants. Development 121, 15191532.

Siegfried, K. R., Eshed, Y., Baum, S. F., Otsuga, D., Drews, G. N. and Bowman, J. L. (1999). Members of the $Y A B B Y$ gene family specify abaxial cell fate in Arabidopsis. Development 126, 4117-4128.

Smith, D. L. and Fedoroff, N. V. (1995). LRP1, a gene expressed in lateral and adventitious root primordia of Arabidopsis. Plant Cell 7, 735-745.

Smyth, D. R., Bowman, J. L. and Meyerowitz, E. M. (1990). Early flower development in Arabidopsis. Plant Cell 2, 755-767.

Tissier, A. F., Marillonnet, S., Klimyuk, V., Patel, K., Torres, M. A., Murphy, G. and Jones, J. D. G. (1999). Multiple independent defective Suppressor-mutator transposon insertions in Arabidopsis: a tool for functional genomics. Plant Cell 11, 1841-1852.

Toori, K. U., Mitsukawa, N., Oosumi, T., Matsuura, Y. and Yokoyama, R. (1996). The Arabidopsis ERECTA gene encodes a putative receptor protein kinase with extracellular leucine-rich repeats. Plant Cell 8, 735-746. 